Bull. Austral. Math. Soc.

$46 \mathrm{~L} 40,47 \mathrm{~B} 47$

Vol. $42(1990)$ [115-120]

\title{
MORE PROPERTIES OF THE PRODUCT OF TWO DERIVATIONS OF A $C^{*}$-ALGEBRA
}

\author{
Martin Mathieu
}

\begin{abstract}
It is proved that the product of two non-zero derivations of a prime $C^{*}$-algebra is bounded only if both of them are bounded.
\end{abstract}

Posner's paper [7] from 1957 initiated the investigation of properties of the product of two derivations, defined on a ring (with additional properties) and, more recently, also in the context of Banach algebras [1], [4], [6]. The so-called first Posner's theorem stating that the product of two non-zero derivations of a prime ring of characteristic different from 2 can never be a derivation has various applications in ring theory, and a number of authors studied possible extensions of this result. We refer to Lanski's paper [2] for a detailed up-to-date discussion of the history and a more comprehensive bibliography.

In [4] we extended Posner's theorem to the case of $C^{*}$-algebra s. This was refined subsequently by Brešar [1] by estimating the distance of the product of two derivations to the set of all derivations, and in [6] an extension to unbounded derivations is given. We also studied compactness properties of $\delta_{1} \delta_{2}$, where $\delta_{1}, \delta_{2}$ are non-zero derivations defined on a $C^{*}$-algebra $A$. For example, $\delta_{1} \delta_{2}$ is weakly compact only if either $\delta_{1}$ or $\delta_{2}$ is weakly compact, and is compact only if both of them are weakly compact, provided $A$ is prime [4, Lemmas 4 and 7]. During a lecture on that paper, J. Cuntz asked whether it is possible to characterise when the product of two densely defined derivations of a $C^{*}$-algebra is a bounded operator. It is the purpose of this note to answer his question. In particular, we prove that the square of a derivation $\delta$ of any $C^{*}$-algebra is bounded only if $\delta$ is bounded (Theorem 1 ) and that the product of two derivations of a prime $C^{*}$-algebra, both non-zero on a dense common core, is bounded only if both of them are bounded (Theorem 2 ). The case of an arbitrary $C^{*}$-algebra can then be deduced easily from Theorem 2 .

Let us fix our notation. Throughout, $A$ will denote a $C^{*}$-algebra and $\delta$ a derivation of $A$ defined on $\mathcal{D}(\delta)$, a subalgebra of $A$. By $L_{a}, R_{b}$, where $a, b \in A$, we understand the left (respectively right) multiplication by $a$ (respectively $b$ ), and $M_{a, b}$ denotes the

Received 14th September 1989

Copyright Clearance Centre, Inc. Serial-fee code: 0004-9729/90 \$A2.00+0.00. 
product $L_{a} R_{b}$. Note that $\left\|M_{a, b}\right\|=\|a\|\|b\|$ if and only if $A$ is a prime $C^{*}$-algebra [3, Proposition 2.3].

We first study the square of a densely defined derivation since the arguments involved are more direct than (although related to) those of Theorem 2. In all algebraic manipulations with unbounded operators that follow we tacitly assume that their domains fit together appropriately.

Theorem 1. Let $\delta$ be a densely defined derivation of a $C^{*}$-algebra $A$. If $\delta^{2}$ is bounded, then $\delta$ itself is bounded.

Proof: For all $x, y \in \mathcal{D}(\delta)$ we have

$$
2 \delta(x) y \delta(x)=\delta^{2}(x y x)-\delta^{2}(x y) x-x \delta^{2}(y x)+x \delta^{2}(y) x .
$$

Hence, $2\|\delta(x) y \delta(x)\| \leqslant 4\left\|\delta^{2}\right\|\|x\|^{2}\|y\|$ whence

$$
\begin{aligned}
\|\delta(x)\|^{2} & =\left\|M_{\delta(x), \delta(x)}\right\|=\sup \{\|\delta(x) y \delta(x)\| \mid\|y\|=1\} \\
& \leqslant 2\left\|\delta^{2}\right\|\|x\|^{2} .
\end{aligned}
$$

Therefore, $\sup \{\|\delta(x)\| \mid\|x\|=1, x \in \mathcal{D}(\delta)\} \leqslant \sqrt{2\left\|\delta^{2}\right\|}$ and $\delta$ is a bounded operator on $\mathcal{D}(\delta)$ which, by the density, can be extended to $A$.

REMARK. The resulting norm estimate $\|\delta\|^{2} / 2 \leqslant\left\|\delta^{2}\right\| \leqslant\|\delta\|^{2}$ cannot be improved in general, as can be seen by an example in [1].

We prepare the proof of Theorem 2 by two auxiliary results, the first of which having an obvious extension to $n$-linear mappings.

Lemma 1. Let $A$ be a prime $C^{*}$-algebra. For $a, b, c \in A$ let $M_{a, b, c}$ : $A \times A \rightarrow A$ denote the bilinear mapping $(z, w) \mapsto a z b w c$. Then $\left\|M_{a, b, c}\right\|=\|a\|\|b\|\|c\|$.

Proof: By definition,

$$
\begin{aligned}
\left\|M_{a, b, c}\right\| & =\sup _{\|z\|=1} \sup _{\|w\|=1}\|a z b w c\|=\sup _{\|z\|=1}\left\|M_{a z b, c}\right\| \\
& =\sup _{\|z\|=1}\|a z b\|\|c\|=\left\|M_{a, b}\right\|\|c\|=\|a\|\|b\|\|c\|,
\end{aligned}
$$

where we used [3, Proposition 2.3] twice.

In [4], we conjectured that the norm of $M_{a, b}+M_{b, a}$ is not less than $\|a\|\|b\|$, provided that $A$ is a prime $C^{*}$-algebra. We do not know at present whether the slightly smaller estimate given in the next lemma is sharp.

Lemma 2. Let $A$ be a prime $C^{*}$-algebra and $a, a^{\prime}, b, b^{\prime}, b^{\prime \prime}, c \in A$. Then

$$
\begin{aligned}
2\|a\|\|b\|\|c\| \leqslant & \left\|M_{a, b}+M_{b^{\prime}, a^{\prime}}\right\|\|c\| \\
& +\left\|M_{b, c}+M_{a^{\prime}, b^{\prime \prime}}\right\|\|a\|+\left\|M_{a, b^{\prime \prime}}+M_{b^{\prime}, c}\right\|\left\|a^{\prime}\right\| .
\end{aligned}
$$


In particular, $\left\|M_{a, b}+M_{b, a}\right\| \geqslant 2\|a\|\|b\| / 3$.

Proof: The identity

$$
2 a z b w c=\left(a z b+b^{\prime} z a^{\prime}\right) w c+a z\left(b w c+a^{\prime} w b^{\prime \prime}\right)-a\left(z a^{\prime} w\right) b^{\prime \prime}-b^{\prime}\left(z a^{\prime} w\right) c,
$$

holding for all $z, w \in A$, yields

$$
\begin{aligned}
2\|a z b w c\| \leqslant \| M_{a, b} & +M_{b^{\prime}, a^{\prime}}\|\| z\|\| w\|\| c \| \\
& +\left\|M_{b, c}+M_{a^{\prime}, b^{\prime \prime}}\right\|\|z\|\|w\|\|a\|+\left\|M_{a, b^{\prime \prime}}+M_{b^{\prime}, c}\right\|\|z\|\|w\|\left\|a^{\prime}\right\| .
\end{aligned}
$$

Hence, Lemma 1 entails the first assertion, and specialising to $a=a^{\prime}=c$ and $b=b^{\prime}=$ $b^{\prime \prime}$ gives the second.

Next is the main result of the paper.

THEOREM 2. Let $\delta_{1}, \delta_{2}$ be $t$ wo derivations of a prime $C^{*}$-algebra $A$ that are non-zero on $\mathcal{D}\left(\delta_{1}\right) \cap \mathcal{D}\left(\delta_{2}\right)$ which is dense. Then $\delta_{1} \delta_{2}$ is bounded if and only if both $\left(\delta_{1}\right)$ and $\left(\delta_{2}\right)$ are bounded.

Proof: Applying Lemma 2 with $x, y, u \in \mathcal{D}=\mathcal{D}\left(\delta_{1}\right) \cap \mathcal{D}\left(\delta_{2}\right)$ we have

$$
\begin{aligned}
2\left\|\delta_{1}(x)\right\|\left\|\delta_{2}(y)\right\|\left\|\delta_{1}(u)\right\| & \leqslant\left\|M_{\delta_{1}(x), \delta_{2}(y)}+M_{\delta_{2}(x), \delta_{1}(y)}\right\|\left\|\delta_{1}(u)\right\| \\
& +\| M_{\delta_{2}(y), \delta_{1}(u)}+M_{\delta_{1}(y), \delta_{2}(u)\|\| \delta_{1}(x) \|} \\
& +\left\|M_{\delta_{1}(x), \delta_{2}(u)}+M_{\delta_{2}(x), \delta_{1}(u)}\right\|\left\|\delta_{1}(y)\right\| .
\end{aligned}
$$

Suppose that $\delta_{1} \delta_{2}$ is bounded. A straightforward calculation yields

$$
M_{\delta_{1}(x), \delta_{2}(y)}+M_{\delta_{2}(x), \delta_{1}(y)}=\delta_{1} \delta_{2} M_{x, y}-R_{y} \delta_{1} \delta_{2} L_{x}-L_{x} \delta_{1} \delta_{2} R_{y}+M_{x, y} \delta_{1} \delta_{2}
$$

(compare also [1, Observation 1]). Combining (1) and (2) we thus obtain

$$
\begin{aligned}
2\left\|\delta_{1}(x)\right\|\left\|\delta_{2}(y)\right\|\left\|\delta_{1}(u)\right\| \leqslant & 4\left\|\delta_{1} \delta_{2}\right\|\left(\|x\|\|y\|\left\|\delta_{1}(u)\right\|\right. \\
& \left.+\|y\|\|u\|\left\|\delta_{1}(x)\right\|+\|x\|\|u\|\left\|\delta_{1}(y)\right\|\right) .
\end{aligned}
$$

Now fix $x$ and $u$ such that $\|x\|=\|u\|=1$ and $\delta_{1}(x) \neq 0, \delta_{1}(u) \neq 0$. Then, for each $y \in \mathcal{D}$ with $\|y\|=1$,

$$
\begin{gathered}
\left\|\delta_{2}(y)\right\| \leqslant \gamma_{1}+\gamma_{2}\left\|\delta_{1}(y)\right\| \\
\gamma_{1}=\left(2\left\|\delta_{1} \delta_{2}\right\|\left(\left\|\delta_{1}(x)\right\|+\left\|\delta_{1}(u)\right\|\right)\right) /\left(\left\|\delta_{1}(x)\right\|\left\|\delta_{1}(u)\right\|\right) \\
\gamma_{2}=\left(2\left\|\delta_{1} \delta_{2}\right\|\right) /\left(\left\|\delta_{1}(x)\right\|\left\|\delta_{1}(u)\right\|\right)
\end{gathered}
$$

where

and 
are greater than zero by (3). If there is a sequence $\left(y_{n}\right)_{n \in N}$ in $\mathcal{D}$ such that $\left\|y_{n}\right\|=1$ and $\left\|\delta_{2}\left(y_{n}\right)\right\|=n$ for all $n \in N$, then $\left\|\delta_{1}\left(y_{n}\right)\right\|$ increases too, by (4). However, by Lemma 2 and (2),

$$
\frac{2}{3}\left\|\delta_{1}(y)\right\|\left\|\delta_{2}(y)\right\| \leqslant\left\|M_{\delta_{1}(y), \delta_{2}(y)}+M_{\delta_{2}(y), \delta_{1}(y)}\right\| \leqslant 4\left\|\delta_{1} \delta_{2}\right\|
$$

for every $y \in \mathcal{D}$ with $\|y\|=1$, which shows that $\delta_{2}$ has to be bounded on $\mathcal{D}$ and can thus be extended to $A$.

Interchanging $\delta_{1}$ with $\delta_{2}$ in (1) yields

$$
\begin{aligned}
\left\|\delta_{2}(x)\right\|\left\|\delta_{1}(y)\right\|\left\|\delta_{2}(u)\right\| & \leqslant 2\left\|\delta_{1} \delta_{2}\right\|\left(\|x\|\|y\|\left\|\delta_{2}(u)\right\|\right. \\
& \left.+\|y\|\|u\|\left\|\delta_{2}(x)\right\|+\|x\|\|u\|\left\|\delta_{2}(y)\right\|\right)
\end{aligned}
$$

whence

$$
\sup \left\{\left\|\delta_{1}(y)\right\| \mid y \in \mathcal{D},\|y\|=1\right\}\left\|\delta_{2}\right\|^{2} \leqslant 6\left\|\delta_{1} \delta_{2}\right\|\left\|\delta_{2}\right\| \text {. }
$$

Since $\delta_{2}$ is non-zero it follows that $\delta_{1}$ is bounded (by $\left.\left(6\left\|\delta_{1} \delta_{2}\right\|\right) /\left\|\delta_{2}\right\|\right)$, and the proof is complete.

REMarks: 1. Observe that both Theorems 1 and 2 remain valid under the assumption that $A$ is a Banach algebra which is ultraprime, that is $\inf \left\{\left\|M_{a, b}\right\| \mid a, b \in\right.$ $A,\|a\|=\|b\|=1\}>0$, in particular, if $A$ is the algebra of all bounded operators on a Banach space.

2. Let $A$ be a prime $C^{*}$-algebra, and let $\delta_{1}, \delta_{2}$ be derivations of $A$ such that $\delta_{1} \delta_{2}$ is again a derivation and $\mathcal{D}=\mathcal{D}\left(\delta_{1}\right) \cap \mathcal{D}\left(\delta_{2}\right) \cap \mathcal{D}\left(\delta_{1} \delta_{2}\right)$ is a dense common core. Then a well known calculation (see [7, p. 1094]) shows that $M_{\delta_{1}(x), \delta_{2}(y)}+M_{\delta_{2}(x), \delta_{1}(y)}=0$ for all $x, y \in \mathcal{D}$. By (1), it follows that $\left\|\delta_{1}(x)\right\|^{2}\left\|\delta_{2}(y)\right\|=0$ for all $x, y \in \mathcal{D}$, thus either $\delta_{1}=0$ or $\delta_{2}=0$. We thus obtain Posner's theorem for unbounded derivations more directly than in [6].

Now suppose that $\delta$ is an unbounded derivation of a $C^{*}$-algebra $B$. Putting $A=B \oplus B, \delta_{1}=\delta \oplus 0$ and $\delta_{2}=0 \oplus \delta$ we obtain two unbounded derivations with bounded product on a non-prime $C^{*}$-algebra. Applying Theorem 2 in irreducible representations of an arbitrary $C^{*}$-algebra, we proceed to show that this example is the only exceptional case which can occur.

Corollary 1. Let $\delta_{1}, \delta_{2}$ be two derivations of a $C^{*}$-algebra $A$ such that $\mathcal{D}=$ $\mathcal{D}\left(\delta_{1}\right) \cap \mathcal{D}\left(\delta_{2}\right)$ is dense and $\delta_{1} \delta_{2}$ is bounded. For every irreducible representation $\pi$, if $\pi \delta_{i}$ is non-zero on $\mathcal{D}$, then $\pi \delta_{j}$ is bounded where $1 \leqslant i, j \leqslant 2, i \neq j$.

Proof: Let $(\pi, H)$ be an irreducible representation of $A$. Since, by Kaplansky's density theorem, the canonical extension $\tilde{\pi}$ of $\pi$ to the enveloping von Neumann algebra $A^{* *}$ maps the unit ball of $A^{* *}$ onto the unit ball of $\pi(A)^{\prime \prime}=B(H)[8$, III.2.2], for 
every $a, b, c, d \in A$, we have $\left\|M_{\pi(a), \pi(b)}+M_{\pi(c), \pi(d)}\right\| \leqslant\left\|M_{a, b}+M_{c, d}\right\|$. An application of $\pi$ to (1) together with (2) gives

$$
\begin{aligned}
\left\|\pi \delta_{1}(x)\right\|\left\|\pi \delta_{2}(y)\right\|\left\|\pi \delta_{1}(u)\right\| \leqslant & 2\left\|\delta_{1} \delta_{2}\right\|\left(\|x\|\|y\|\left\|\pi \delta_{1}(u)\right\|\right. \\
& \left.+\|y\|\|u\|\left\|\pi \delta_{1}(x)\right\|+\|x\|\|u\|\left\|\pi \delta_{1}(y)\right\|\right)
\end{aligned}
$$

whenever $x, y, u \in \mathcal{D}$. If $\pi \delta_{1}$ is non-zero on $\mathcal{D}$, we can continue as in the proof of Theorem 2 and conclude that $\pi \delta_{2}$ has to be bounded. Working with the analogue of $\left(3^{\prime}\right)$ rather than (3), we deduce the boundedness of $\pi \delta_{1}$ from $\pi \delta_{2}$ being non-zero.

Observe also that both $\pi \delta_{1}$ and $\pi \delta_{2}$ non-zero implies that $\pi \delta_{1} \delta_{2}$ is non-zero. This is derived either from the above arguments or from [3, Theorem 4.1] applied to the identity

$$
M_{\pi \delta_{1}(x), \pi \delta_{2}(y)}+M_{\pi \delta_{2}(x), \pi \delta_{1}(y)}=0 \text { for all } x, y \in \mathcal{D}
$$

which holds if $\pi \delta_{1} \delta_{2}=0$.

The information obtained in Theorem 2 can also be assembled to a global picture, if we allow scaling by central elements in $A^{* *}$. Take a faithful family $\Gamma$ of disjoint irreducible representations of $A$, put $\Gamma_{0}=\left\{\pi \in \Gamma \mid \pi \delta_{1}\right.$ and $\pi \delta_{2}$ are non-zero on $\left.\mathcal{D}\right\}$ and let $1-e_{3}$ be the central cover of $\Gamma_{0}$ in $A^{* *}$ (see [5, 3.8]). For each $\pi \in \Gamma_{0}$, $\left\|\pi \delta_{1}\right\|\left\|\pi \delta_{2}\right\| \leqslant 6\left\|\delta_{1} \delta_{2}\right\|$. Put $\Gamma_{1}=\left\{\pi \in \Gamma_{0} \mid\left\|\pi \delta_{1}\right\| \leqslant 1\right\}, \Gamma_{2}=\Gamma_{0} \backslash \Gamma_{1}$, and let $e_{1}, e_{2}$ be the central covers of $\Gamma_{1}, \Gamma_{2}$, respectively. Then, $e_{1} \delta_{1}$ is bounded (by 1) and $c_{1} \delta_{2}$ is bounded (by $6\left\|\delta_{1} \delta_{2}\right\|$ ), where $c_{1}=c_{1} e_{1}=\sum_{\pi \in \Gamma_{1}} \oplus\left\|\pi \delta_{1}\right\| c(\pi), c(\pi)$ is the central cover of $\pi$, and $c \delta=L_{c} \delta$ for each central element $c$ in $A^{* *}$. In the same vein, $e_{2} \delta_{2}$ is bounded (by $6\left\|\delta_{1} \delta_{2}\right\|$, since $\inf \left\{\left\|\pi \delta_{1}\right\| \mid c(\pi) \leqslant e_{2}\right\} \geqslant 1$ ) and $c_{2} \delta_{1}$ is bounded (by $\left.6\left\|\delta_{1} \delta_{2}\right\|\right)$, where $c_{2}=c_{2} e_{2}=\sum_{\pi \in \Gamma_{2}} \oplus\left\|\pi \delta_{2}\right\| c(\pi)$. (Observe that $\mathrm{c}(\pi) \delta=\pi \delta$.)

Summarising this we have deduced our final result.

Corollary 2. Let $\delta_{1}, \delta_{2}$ be two derivations of a $C^{*}$-algebra $A$ such that $\mathcal{D}\left(\delta_{1}\right) \cap \mathcal{D}\left(\delta_{2}\right)$ is dense. If $\delta_{1} \delta_{2}$ is bounded then there exist orthogonal central projections $e_{j} \in A^{* *}, j=1,2,3$, with $e_{1}+e_{2}+e_{3}=1$ and central elements $c_{i} \in A^{* *}, i=1,2$, such that $e_{i} \delta_{i}$ and $c_{j} \delta_{i}$ are bounded for $1 \leqslant i, j \leqslant 2, i \neq j$, and $e_{3} \delta_{i}$ have orthogonal ranges.

\section{REFERENCES}

[1] M. Brešar, 'On the composition of two derivations in certain normed algebras', (preprint, June 1989).

[2] C. Lanski, 'Differential identities, Lie ideals, and Posner's theorems', Pacific J. Math. 134 (1988), 275-297. 
[3] M. Mathieu, 'Elementary operators on prime C*-algebras I', Math. Ann. 284 (1989), 223-244.

[4] M. Mathieu, 'Properties of the product of two derivations of a $\mathrm{C}^{*}$-algebra', Canad. Math. Bull. 32 (1989), 490-497.

[5] G. K. Pedersen, $C^{*}$-algebras and their automorphism groups (Academic Press, London, 1979).

[6] S. Pedersen, 'The product of two (unbounded) derivations', (preprint, June 1989).

[6] E. C. Posner, 'Derivations in prime rings', Proc. Amer. Math. Soc. 8 (1957), 1093-1100.

[7] M. Takesaki, Theory of operator algebras I (Springer-Verlag, New York - Heidelberg Berlin, 1979).

Mathematisches Institut der Universitãt Tübingen

Auf der Morgenstelle 10

D-7400 Tübingen

Federal Republic of Germany 